\title{
The single-nucleotide polymorphism 309 in the MDM2 gene contributes to the Li-Fraumeni syndrome and related phenotypes
}

\author{
Mariëlle WG Ruijs ${ }^{1,2}$, Marjanka K Schmidt ${ }^{3}$, Heli Nevanlinna ${ }^{4}$, Johanna Tommiska ${ }^{4}$, \\ Kristiina Aittomäki ${ }^{5}$, Roelof Pruntel ${ }^{1}$, Senno Verhoef ${ }^{1}$ and Laura J van`t Veer ${ }^{*}, 1$
}

\begin{abstract}
${ }^{1}$ Family Cancer Clinic, The Netherlands Cancer Institute, Amsterdam, The Netherlands; ${ }^{2}$ Department of Clinical Genetics and Human Genetics, VU University Medical Center, Amsterdam, The Netherlands; ${ }^{3}$ Department of Epidemiology, The Netherlands Cancer Institute, Amsterdam, The Netherlands; ${ }^{4}$ Department of Obstetrics and Gynaecology, Helsinki University Central Hospital, Helsinki, Finland; ${ }^{5}$ Department of Clinical Genetics, Helsinki University Central Hospital, Helsinki, Finland
\end{abstract}

Li-Fraumeni syndrome (LFS) is an autosomal-dominant cancer predisposition syndrome of which the majority is caused by TP53 germline mutations and is characterised by different tumour types occurring at relatively young age. Recently, it was shown that a single-nucleotide polymorphism (SNP) in the MDM2 gene, SNP309 (T>G variation), was associated with accelerated tumour formation in LFS patients who carry a TP53 germline mutation. To confirm this finding in different populations, we screened 25 Dutch and 11 Finnish TP53 mutation carriers for the presence of the SNP309 G allele in the MDM2 gene.

Additionally, we investigated whether the SNP309 G allele plays a role in 72 Dutch TP53-negative LFS and LFS-related patients. In the TP53 germline mutation carriers, a significant difference was seen in the mean age of tumour onset for the SNP309 G allele group, that is, 29.7 years as compared to the SNP309 homozygous T group 45.5 years $(P=\mathbf{0 . 0 0 5})$. In patients of LFS and LFS-related TP53-negative families, no difference was seen in the mean age of tumour onset. However, this TP53-negative group did show a significantly higher percentage of SNP309 homozygotes $(G / G)$ compared to the general population $(P=0.02)$. In conclusion, TP53 germline mutation carriers who have an SNP309 $G$ allele have an earlier onset of tumour formation. The higher prevalence of MDM2 SNP309 homozygous G/G carriers in the TP53negative group suggests that this allele contributes to cancer susceptibility in LFS and LFS-related families. European Journal of Human Genetics (2007) 15, 110-114. doi:10.1038/sj.ejhg.5201715; published online 27 September 2006

Keywords: Li-Fraumeni syndrome; TP53; MDM2; single-nucleotide polymorphism; SNP309

Introduction

$\mathrm{Li}$-Fraumeni syndrome (LFS) is an autosomal-dominant cancer predisposition syndrome. The main tumour types of LFS include bone- and soft-tissue sarcoma (STS), breast

*Correspondence: Dr LJ van't Veer, Department of Pathology, Netherlands Cancer Institute, Plesmanlaan 121, Amsterdam 1066CX, The Netherlands. Tel: +31 20 5122754; Fax: +31 20 5122759;

E-mail: I.vt.veer@nki.nl

Received 16 February 2006; revised 24 May 2006; accepted 11 July 2006; published online 27 September 2006 cancer, brain tumour, adrenocortical carcinoma and leukaemia. ${ }^{1}$ The classical LFS criteria are a proband with a sarcoma aged under 45 years and a first-degree relative with any cancer aged under 45 years, plus a first- or seconddegree relative in the same lineage with any cancer aged under 45 years or a sarcoma at any age. ${ }^{2}$ In addition, LiFraumeni-like syndrome (LFL) criteria were formulated as a proband with any childhood tumour, or a sarcoma, brain tumour or adrenocortical tumour aged under 45 years and a first or second degree in the same lineage with a typical LFS tumour at any age, plus a first- or second-degree 
relative in the same lineage with any cancer under the age of 60 years. ${ }^{3}$ Less stringent LFL criteria were formulated by Eeles $^{4}$ as two first- or second-degree relatives with typical LFS tumours at any age. In 1990, germline mutations in the TP53 gene were found to be associated with LFS. $^{5}$ At present, TP53 germline mutations are detected in approximately $75 \%$ of LFS and $40 \%$ of LFL families. ${ }^{6}$ Because still $25-60 \%$ of LFS/LFL families do not carry a germline TP53 mutation, alternative LFS susceptibility genes have been proposed. $^{7}$ Moreover, single-nucleotide polymorphisms (SNP) may contribute to predisposition to cancer. ${ }^{8}$ Recently, it was suggested that an SNP in the promoter region of the MDM2 gene is associated with a significantly earlier age of onset of tumours in both TP53 mutation carriers and sporadic TP53-negative STS. ${ }^{9}$ The MDM2 gene is an important negative regulator of TP53. Bond et al ${ }^{9}$ showed that the MDM2 SNP309 G creates an improved SP1 site, leading to higher basal levels of MDM2 in cells, thereby attenuating the p53 pathway. The impact of the MDM2 SNP309 G on the age of tumour onset in germline TP53 mutation carriers was confirmed by Bougeard et $a l,{ }^{10}$ by screening 41 affected TP53 mutation carriers for the presence of an SNP309 G allele. Wilkening et al ${ }^{11}$ showed that this polymorphism does not seem to play a role in familial breast cancer.

We investigated the presence of the SNP309 G allele in Dutch and Finnish TP53 mutation carriers to look at the effect on age of onset. Furthermore, we tested 72 family members of 68 TP53-negative LFS and LFS-related families from the Netherlands to determine the possible effect of SNP309 G on cancer susceptibility.

\section{Patients and methods}

For this study, 108 selected cancer patients were screened for TP53 germline mutations, 97 at the Family Cancer Clinic of the Netherlands Cancer Institute and 11 at the Helsinki University Central Hospital. These patients were divided into four groups: (1) classic LFS, ${ }^{2}$ (2) LFL according to Birch et $a l^{3}$ or Eeles, ${ }^{4}$ (3) LFS-suggestive, including childhood onset (under 18 years) sarcoma or brain tumours, two or more primary tumours at any age, or two first-degree relatives with a tumour at any age, of which at least one is a typical LFS tumour and (4) breast cancer before 30 years of age (without detectable BRCA 1 or BRCA2 mutation) (Table 1). Groups 2-4 combined are further referred to as LFS-related families. Thirty-six TP53positive and 72 TP53-negative family members out of 87 families (19 TP53-positive families, 68 TP53-negative families, respectively) were tested for the presence of SNP309 $\mathrm{G}$ in the MDM2 gene. The 68 TP53-negative families were all Dutch; in Finland, TP53-negative families were not tested for the presence of SNP309 G in the MDM2 gene. DNA from peripheral blood lymphocytes was isolated

Table 1 SNP309 genotype in patients and controls

(a)

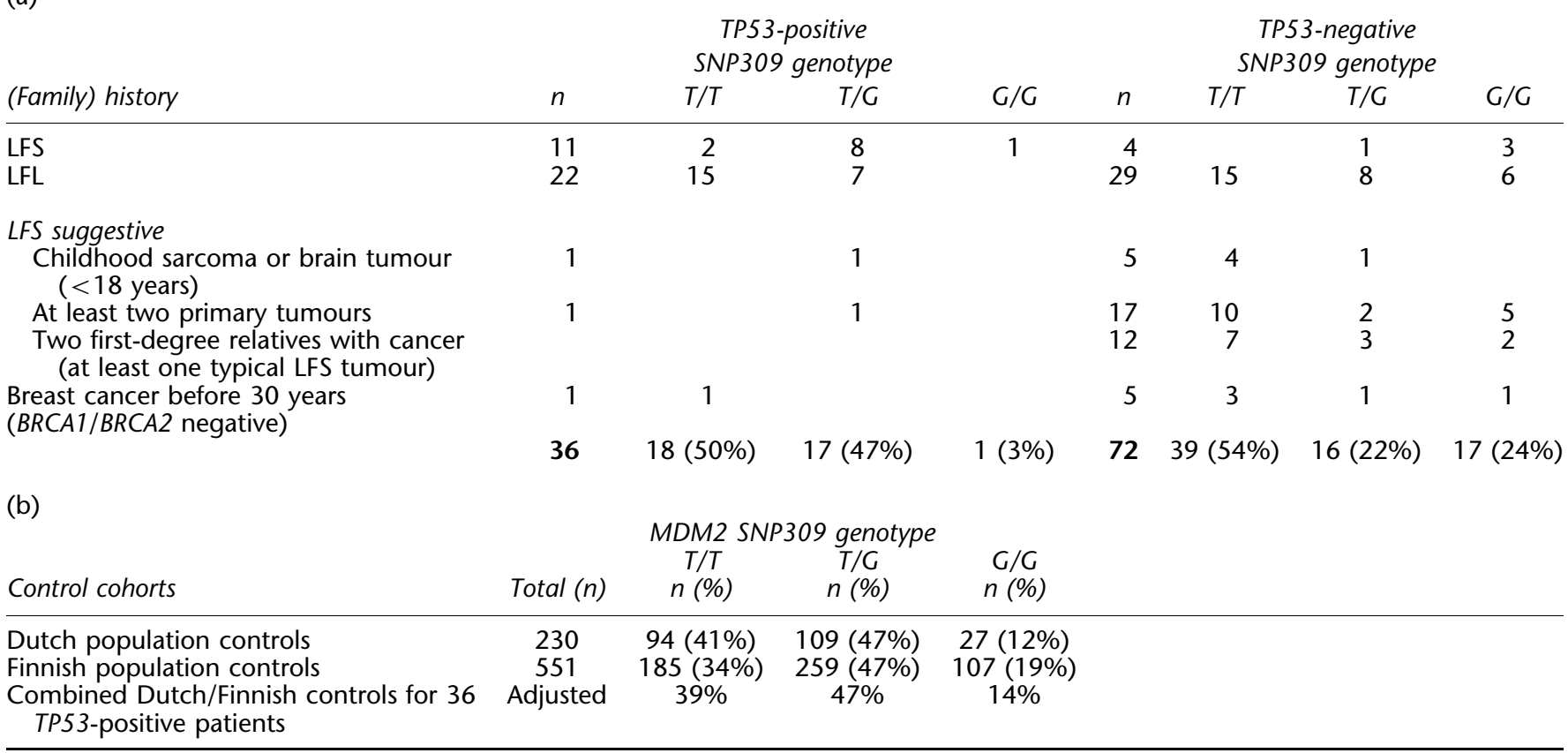

(a) SNP309 genotype in TP53-positive and TP53-negative patients. Patients were divided into four groups according to their (family) history.

(b) SNP309 genotype in Dutch and Finnish controls.

$\mathrm{LFS}=\mathrm{Li}$-Fraumeni syndrome fulfilling the classical criteria, ${ }^{2} \mathrm{LFL}=\mathrm{Li}-$ Fraumeni-like syndrome according to Birch ${ }^{3}$ or Eeles. $^{4}$ 
according to standard procedures. Mutation analysis of the TP53 gene was performed by sequence analysis of all coding exons (2-11) and flanking intron-exon boundaries of these exons using standard procedures. The 19 TP53 germline mutations in the TP53-positive families included 12 missense mutations, four stop mutations and three splice site mutations. DNA was sequenced for the presence of SNP309 G in the MDM2 promoter (Different primer sets were used in the Netherlands and Finland; primer 1: $5^{\prime}$ TGTAAAACGACGGCCAGTCGGGAGTTCAGGGTAAAGGT-3', primer 2: 5'-CAGGAAACAGCTATGACCTCGGAACGTGT CTGAACTTG-3' in Amsterdam, primer 1: 5'-GTTTTGTTGG ACTGGGGCTA-3', primer 2: 5'-CGGAACGTGTCTGAAC TTGA-3' in Helsinki).

A control group of 230 Dutch healthy blood bank controls, males and females and 551 Finnish healthy female control subjects (from the same geographical region) were screened for the presence of SNP309 G. Proportions of haplotype frequencies in the combined control group were adjusted for the numbers of Dutch and Finnish patients. The Dutch controls were genotyped as described above, the Finnish controls were genotyped using restriction fragment length polymorphisms: PCR products were digested with MspA1 I (New England Biolabs) and run on a $3 \%$ agarose gel; the PCR product with a $\mathrm{T}$ allele is cut once and the PCR product with a G allele is cut twice.

A $t$-test was used to determine the statistical significance for the age of onset of cancer between the groups with and without SNP309 G. Proportions were tested by a $\chi^{2}$ test.

\section{Results}

The classification of the 108 LFS and LFS-related patients into four groups (see Patients and methods) is shown in Table 1a. The presence of the SNP309 G in TP53-positive patients was found in heterozygous state, T/G, in 17 out of $36(47 \%)$ patients and in the homozygous state, G/G, in one out of $36(3 \%)$ patients. In TP53-negative patients, $\mathrm{T} / \mathrm{G}$ was found in 16 out of 72 (22\%) patients, and G/G was found in 17 out of 72 patients (24\%, Table 1a). The Dutch and Finnish population controls, including the ratioadjusted Dutch/Finnish controls are listed in Table 1b. In the TP53-negative LFS and LFS-related patients, the G/G genotype was twice the percentage in the general population (24 vs 12\%, respectively, $P=0.02$ ), and the $\mathrm{T} / \mathrm{G}$ genotype proportion was significantly lower as compared to the general population ( 22 vs $47 \%, P<0.01$ ).

The prevalence of the different first tumour types in both TP53-positive and TP53-negative patients is shown in Figure $1 \mathrm{a}$ and $\mathrm{b}$, with breast cancer and STS being the most frequent cancers. The average age of onset of all first cancers was 34.3 years. When the group is divided in TP53positive and TP53-negative patients, the average age was
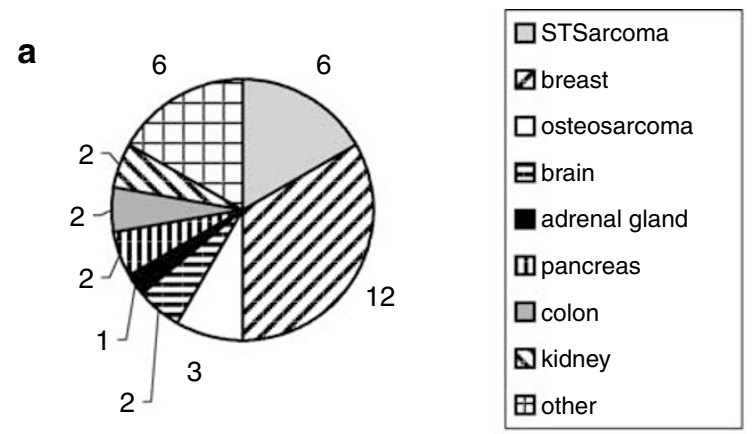

b

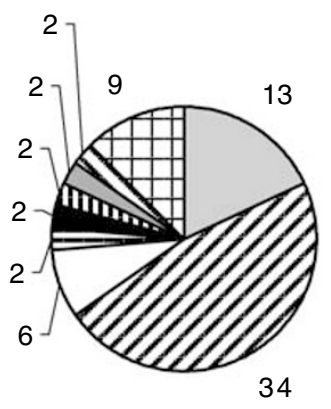

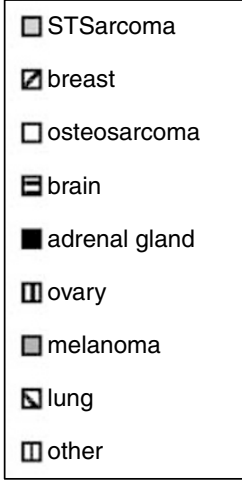

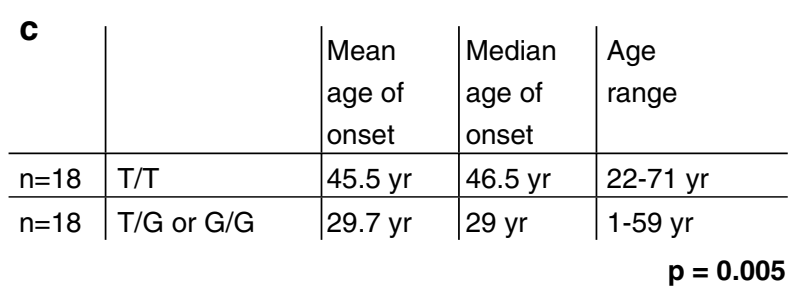

\begin{tabular}{l|l|l|l|l}
$\mathbf{d}$ & & $\begin{array}{l}\text { Mean } \\
\text { age of } \\
\text { onset }\end{array}$ & $\begin{array}{l}\text { Median } \\
\text { age of } \\
\text { onset }\end{array}$ & $\begin{array}{l}\text { Age } \\
\text { range }\end{array}$ \\
\hline $\mathrm{n}=39$ & $\mathrm{~T} / \mathrm{T}$ & $32.1 \mathrm{yr}$ & $36 \mathrm{yr}$ & $0.5-64 \mathrm{yr}$ \\
\hline $\mathrm{n}=33$ & $\mathrm{~T} / \mathrm{G}$ or $\mathrm{G} / \mathrm{G}$ & $33.3 \mathrm{yr}$ & $32 \mathrm{yr}$ & $0.5-63 \mathrm{yr}$ \\
$\mathbf{p = 0 . 7 4 9}$
\end{tabular}

Figure 1 Prevalence of different tumour types and mean age of tumour onset. (a) First tumours in TP53 mutation carriers $(n=36)$. Other = stomach cancer, melanoma, mesothelioma, ovary cancer, lung cancer and thyroid cancer. (b) First tumours in TP53-negative LFS and LFS-related patients $(n=72)$. Other = bladder cancer, Hodgkin's lymphoma, kidney cancer, thyroid cancer, basal cell carcinoma, acute myeloid leukaemia, colon cancer, non-Hodgkin's lymphoma and stomach cancer. (c) Mean age of tumour onset in TP53 mutation carriers according to their MDM2 SNP309 genotype. (d) Mean age of tumour onset in TP53-negative LFS and LFS-related patients according to their MDM2 SNP309 genotype.

37.6 and 32.6 years, respectively. When the TP53-positive group was further divided by the presence or absence of SNP309 G, the T/T group revealed a mean age of 45.5 years, as compared to the group where SNP309 G was present in 
heterozygous or homozygous state (T/G or $\mathrm{G} / \mathrm{G}$ ) where the mean age was significantly earlier, 29.7 years $(P=0.005$, Figure 1c). In the T/T group of TP53-negative patients, the mean age was 32.1 years, and in the $T / G$ and $G / G$ group combined, the mean age was 33.3 years $(P=0.749$; Figure 1d).

\section{Discussion}

We showed that the presence of an SNP in the MDM2 gene, SNP309 ( $\mathrm{T}>\mathrm{G}$ variation) in Dutch and Finnish TP53 germline mutation carriers was associated with accelerated tumour formation. Our results show a 16 years earlier age of tumour onset in TP53 mutation carriers with an SNP309 G allele as compared to the T/T SNP 309 group (Figure 1c), confirming the conclusions of Bond et $a l^{9}$ and Bougeard et $a l,{ }^{10}$ who showed an age difference of 7 and 10 years, respectively. The SNP309 G polymorphism is proposed to act as a genetic modifying factor in TP53 mutation carriers. Because of the small numbers, our analysis has been carried out in a joint sample of Dutch and Finnish origin. Although the allele frequencies and genotype frequencies differ between the Dutch and Finnish controls, as shown in Table $1 \mathrm{~b}$, the genotype frequencies in the TP53positive group did not significantly differ from the ratioadjusted Dutch/Finnish control group, allowing a combined analysis.

Neither Bond et $a l^{9}$ nor Bougeard et $a l^{10}$ screened LFS and LFS-related patients without a TP53 germline mutation. In our TP53-negative LFS and LFS-related group, the age of tumour onset for SNP $309 \mathrm{G}$ allele patients and T/T patients was not significantly different. The presence of the SNP $309 \mathrm{G}$ allele was therefore not shown to have a tumour accelerating effect in our TP53-negative LFS-related patients. However, it was striking to see that the percentage of the G/G genotype in the TP53-negative LFS and LFS-related patients is twice the percentage in the general population. These data indicate an association between homozygosity for SNP309 $(\mathrm{G} / \mathrm{G})$ and the occurrence of cancer, although not related to the age of onset. The fact that these patients were considered for counselling might well be due, in part, to the presence of the homozygous $G$ allele, under the assumption of the SNP309 G polymorphism acting as a modifier or (additional) disease-causing factor. Within the TP53-negative group, there is no difference in the age of tumour onset in the homozygous SNP309 G carriers as compared to the T/T and T/G carriers. Although three out of four classical LFS family members show a G/G genotype, the family history does not seem to be specific for the homozygous SNP309 G carriers (Table 1).

Bond et $a l^{9}$ found the presence of SNP309 to be associated with earlier age of onset for sporadic STS, specifically for the homozygotes (G/G). In our TP53negative patients, 13 patients developed STS as first tumour, six patients showed a T/T genotype, five a T/G genotype and two a $\mathrm{G} / \mathrm{G}$ genotype, the mean ages did not significantly differ between the $G / G$ and $T / G$ or $T / T$ groups. Although both groups (our group of TP53-negative LFS and LFS-related STS patients and Bond's group of sporadic STS) are TP53 negative, the difference in proportion of G/G and in family history of patients, in addition to the small numbers in both studies, could explain the discordance in age of onset.

In conclusion, our results confirm the association of the presence of SNP309 G with a significantly earlier age of onset of the first tumour in TP53 germline mutation carriers. As such, the TP53 combined MDM2 SNP309 G germline mutation now becomes an established example of how a gene-gene interaction synergistically acts in cancer susceptibility. This association raises the question whether MDM2 SNP309 G analysis should be embedded in clinical diagnosis of TP53 mutation carriers. This remains controversial, especially because for many tumour types no early detection procedures are available.

Although the presence of an SNP309 G allele in TP53negative LFS and LFS-related patients was not shown to be correlated with an earlier age of onset of tumours, the higher prevalence of MDM2 SNP309 homozygous G/G carriers in the TP53-negative group may suggest that this allele contributes to the LFS phenotype. Before clinical genetic implementation, these observations need to be confirmed in a larger series of TP53-negative LFS and LFSrelated patients.

\section{Acknowledgements}

We thank Dr Carl Blomqvist and research nurse Nina Puolakka for their kind help. The work was supported by the Dutch National Genomic Initiative, Cancer Genomics Centre, the Helsinki University Central Hospital Research Fund, Academy of Finland (project 110663), Finnish Cancer Society and the Sigrid Juselius Foundation.

\section{References}

1 Li FP, Fraumeni Jr JF: Soft-tissue sarcomas, breast cancer, and other neoplasms. A familial syndrome? Ann Intern Med 1969; 71: $747-752$.

2 Li FP, Fraumeni Jr JF, Mulvihill JJ et al: A cancer family syndrome in twenty-four kindreds. Cancer Res 1988; 48: 5358-5362.

3 Birch JM, Hartley AL, Tricker KJ et al: Prevalence and diversity of constitutional mutations in the p53 gene among $21 \mathrm{Li}-$ Fraumeni families. Cancer Res 1994; 54: 1298-1304.

4 Eeles RA: Germline mutations in the TP53 gene. Cancer Surv 1995; 25: $101-124$.

5 Malkin D, Li FP, Strong LC et al: Germ line p53 mutations in a familial syndrome of breast cancer, sarcomas, and other neoplasms. Science 1990; 250: 1233-1238.

6 Varley JM: Germline TP53 mutations and Li-Fraumeni syndrome. Hum Mutat 2003; 21: 313-320.

7 Bachinski LL, Olufemi SE, Zhou X et al: Genetic mapping of a third $\mathrm{Li}$-Fraumeni syndrome predisposition locus to human chromosome 1q23. Cancer Res 2005; 65: 427-431.

8 Houlston RS, Peto J: The search for low-penetrance cancer susceptibility alleles. Oncogene 2004; 23: 6471-6476. 
9 Bond GL, Hu W, Bond EE et al: A single nucleotide polymorphism in the MDM2 promoter attenuates the p53 tumor suppressor pathway and accelerates tumor formation in humans. Cell 2004; 119: $591-602$.

10 Bougeard G, Baert-Desurmont S, Tournier I et al: Impact of the MDM2 SNP309 and TP53 Arg72Pro polymorphism on age of

tumour onset in Li-Fraumeni syndrome. J Med Genet 2005; 43: 531-533.

11 Wilkening S, Bermejo JL, Burwinkel B et al: The single nucleotide polymorphism IVS1+309 in mouse double minute 2 does not affect risk of familial breast cancer. Cancer Res 2006; 66: $646-648$. 